\title{
A Leptonic Model of Steady High-Energy Gamma-Ray Emission from Sgr $\mathrm{A}^{*}$
}

\author{
Masaaki Kusunose \\ kusunose@kwansei.ac.jp \\ Department of Physics, School of Science and Technology, Kwansei Gakuin University, \\ Sanda 669-1337, Japan \\ and \\ Fumio Takahara \\ takahara@vega.ess.sci.osaka-u.ac.jp \\ Department of Earth and Space Science, Graduate School of Science, Osaka University, \\ Toyonaka 560-0043, Japan
}

\begin{abstract}
Recent observations of Sgr A* by Fermi and HESS have detected steady $\gamma$ ray emission in the $\mathrm{GeV}$ and $\mathrm{TeV}$ bands. We present a new model to explain the $\mathrm{GeV} \gamma$-ray emission by inverse Compton scattering by nonthermal electrons supplied by the NIR/X-ray flares of Sgr $A^{*}$. The escaping electrons from the flare regions accumulate in a region with a size of $\sim 10^{18} \mathrm{~cm}$ and magnetic fields of $\lesssim 10^{-4} \mathrm{G}$. Those electrons produce $\gamma$-rays by inverse Compton scattering off soft photons emitted by stars and dust around the central black hole. By fitting the $\mathrm{GeV}$ spectrum, we find constraints on the magnetic field and the energy density of optical-UV radiation in the central $1 \mathrm{pc}$ region around the supermassive black hole. While the $\mathrm{GeV}$ spectrum is well fitted by our model, the $\mathrm{TeV} \gamma$-rays, whose spectral index is different from that of the $\mathrm{GeV}$ emission, may be from different sources such as pulsar wind nebulae.
\end{abstract}

Subject headings: black hole physics - Galaxy: center — plasmas — radiation mechanisms: non-thermal 


\section{Introduction}

Sagittarius $A^{*}\left(\operatorname{Sgr} A^{*}\right)$ is located at the center of our Galaxy and harbors a massive black hole (see Melia 2007; Genzel et al. 2010, for review). Recent observations have shown that the distance to $\operatorname{Sgr} \mathrm{A}^{*}$ is $\sim 8 \mathrm{kpc}$ (Eisenhauer et al. 2003) and the black hole mass is $\sim 4 \times 10^{6} M_{\odot}$ (e.g., Ghez et al. 2008; Gillessen et al. 2009a, b). The bolometric luminosity of $\mathrm{Sgr} \mathrm{A}{ }^{*}, L_{\mathrm{bol}} \sim 10^{36} \mathrm{erg} \mathrm{s}^{-1}$, is dominated by radio and the peak in the $\nu F_{\nu}$ representation occurs at $\sim 10^{12} \mathrm{~Hz}($ Zylka et al. 1995; Falcke et al. 1998). In the quiescent state, X-ray (2 $-10 \mathrm{keV}$ ) luminosity is very dim, i.e., $L_{X} \sim 2.4 \times 10^{33} \mathrm{erg} \mathrm{s}^{-1}$ (Dodds-Eden et al. 2009, 2011). However, frequent flares are observed in the X-ray band as well as the near infrared (NIR) band (Dodds-Eden et al. 2009, 2011). In the high energy regime, $\mathrm{TeV} \gamma$-rays have been observed by CANGAROO (Tsuchiva et al. 2004), VERITAS (Kosack et al. 2004), HESS (Rolland \& Hinton 2005; Aharonian et al. 2004, 2006, 2008, 2009), and also by MAGIC (Albert et al. 2006). The HESS source is named HESS H1745-290. Recent observations show that $\mathrm{TeV}$ emission exhibits no time variation (Rolland \& Hinton 2005; Albert et al. 2006; Aharonian et al. 2008). More recent observations by Fermi Large Area Telescope find that $\mathrm{GeV} \gamma$-rays are emitted in the region coinciding with $\mathrm{Sgr} \mathrm{A}^{*}$ (Abdo et al. 2009; Cohen-Tanugi et al. 2009). The source is named 1FGL J1745.6-2900. The observed GeV $\gamma$-rays $>300 \mathrm{MeV}$ are from the region around $\mathrm{Sgr} \mathrm{A}^{*}$. The averaged flux of $\mathrm{GeV} \gamma$-rays is $(324.9 \pm 7.05) \times 10^{-9}$ counts $\mathrm{cm}^{-2} \mathrm{~s}^{-1}$ and there is no statistically significant variability. The spectrum is well fitted by a broken power law with the break energy $E_{\mathrm{br}}=2.0_{-1.0}^{+0.8} \mathrm{GeV}$ and the power law indices $\Gamma_{1}=2.20 \pm 0.04\left(E<E_{\mathrm{br}}\right)$ and $\Gamma_{2}=2.68 \pm 0.05\left(E>E_{\mathrm{br}}\right)$ (Chernyakova et al. 2011).

While the emission in radio through infrared and possibly X-ray bands is explained by emission from radiatively inefficient accretion flow (RIAF) (Yuan et al. 2003) or jets (Falcke \& Markoff 2000) near the central black hole, the emission mechanisms of highenergy radiation are still debated. Before the launch of Fermi, Atoyan \& Dermer (2004) proposed a MHD wind shock model for the TeV emission from Sgr A* The TeV emission observed by HESS was modeled also by Ballantyne et al. (2007) (hadronic model) and Hinton \& Aharonian (2007) (leptonic model). In the model by Atoyan \& Dermer (2004), electrons with $\gamma \lesssim 10^{8}$ scatter photons with $\nu \sim 10^{12} \mathrm{~Hz}$ from RIAF and far-infrared dust radiation. This produces $\mathrm{TeV} \gamma$-rays in the Thomson scattering regime. Hinton \& Aharonian (2007) show that TeV emission is explained by inverse Compton (IC) scattering off infrared (IR) and optical photons in pulsar wind nebula G359.95-0.04. The flux of GeV $\gamma$-rays of these models is smaller than the flux observed by Fermi recently. The contribution of high energy sources in the Galactic center region is reviewed by Crocker et al. (2011). Recently, Chernyakova et al. (2011) proposed a hadronic model to explain the spectrum both in the $\mathrm{TeV}$ and GeV bands, assuming that the sources of HESS and Fermi are coincident. Rela- 
tivistic protons injected by Sgr $\mathrm{A}^{*}$ interact with ambient matter and produces $\gamma$-rays. For example, a constant injection of high-energy protons for $10^{4}$ years reproduces the observed very high energy $\gamma$-ray spectrum. The different spectral shapes in the GeV and TeV bands are owing to the different effective speeds of the protons through the ambient matter.

Although the attenuation of $\mathrm{TeV}$ photons by $e^{+} e^{-}$pair production may change the spectral shape of the $\mathrm{TeV} \gamma$-rays, this is not the case for $\mathrm{Sgr} \mathrm{A}^{*}$. As shown by several authors (Porter \& Strong 2005; Moskalenko et al. 2006; Zhan et al. 2006), the attenuation of TeV photons by $e^{+} e^{-}$production on the Galactic interstellar radiation field is weak for photon energy less than $10 \mathrm{TeV}$ (see also Aharonian \& Neronov 2005).

Because HESS and Fermi do not have enough spatial resolution, the coincidence of both sources, HESS H1745 - 290 and 1FGL J1745.6 - 2900, is not conclusive. In this paper we present another model of the steady $\gamma$-ray emission, focusing on the $\mathrm{GeV}$ emission. We recently proposed a synchrotron blob model to explain the NIR/X-ray flares from Sgr A* (Kusunose \& Takahara 2011). In this model the temporal injection of electrons is assumed to produce flares by synchrotron radiation. The frequency of flare events is high, e.g., the peaks of the light curves occur once a day and four times a day in X-ray and NIR bands, respectively (Baganoff 2003; Eckart et al. 2006; Dodds-Eden et al. 2011; Trap et al. 2011). Nonthermal electrons escape from the flare emission region on timescale $\sim$ several $R / c$, where $R \sim 10^{13} \mathrm{~cm}$ is the size of the flare emission region and $c$ is the speed of light. Away from the flare region escaping electrons are accumulated owing to the ambient magnetic fields and emit radiation through interaction with the magnetic fields and ambient radiation fields emitted by stars and dust. There, away from the central accretion flow, the strength of magnetic field is smaller than that in the accretion flow, and IC scattering becomes a dominant radiative process. The Lorentz factor of nonthermal electrons of the flare model is about $10^{4}$ and the ambient radiation field has a peak at $\nu \sim 10^{15} \mathrm{~Hz}$ (Mezger et al. 1996) in a region $<1.2$ pc. Then it is expected that photons with $\nu \sim 10^{23} \mathrm{~Hz}$ are produced by IC scattering and this is in the GeV band observed by Fermi. In this paper we show numerically that the emission by IC scattering naturally explains the GeV emission from Sgr A*.

We describe our model in Section 2 and show numerical results in Section 3, Finally, we discuss our results in Section 4 .

\section{Emission Model}

We assume that high energy electrons are supplied by the flare events that occur near the central black hole. Although there are various high energy sources such as supernova 
remnants in the Galactic center region, we assume that the injection of high-energy particles from the central region is dominant. A spherical geometry with radius $r_{\gamma}$ is assumed for the high-energy (HE) $\gamma$-ray emission region. We solve the kinetic equations of electrons and photons simultaneously to obtain the spectra of electrons and photons self-consistently. In the following we describe our model in detail.

In a steady state, nonthermal electrons are injected at rate $q_{\text {inj }}(\gamma)$ per unit volume and unit interval of $\gamma$, where $\gamma$ is the electron Lorentz factor, and they escape from the HE emission region on timescale $t_{\mathrm{esc}}$. The kinetic equation of the electrons in a steady state is given by

$$
-\frac{\partial}{\partial \gamma}\left[\dot{\gamma}_{\mathrm{rad}} n_{e}(\gamma)\right]-\frac{n_{e}(\gamma)}{t_{\mathrm{esc}}}+q_{\mathrm{inj}}(\gamma)=0
$$

where $n_{e}(\gamma)$ is the electron number density per unit interval of $\gamma$ and $m_{e} c^{2} \dot{\gamma}_{\text {rad }}(<0)$ is the radiative cooling rate of an electron with $m_{e}$ being the electron mass. The emission mechanisms are synchrotron radiation and IC scattering. Here the soft photon sources are the synchrotron radiation by the nonthermal electrons in the HE emission region (synchrotron self-Compton, or SSC) and the photons emitted by stars and dust (external Compton scattering). The average magnetic field of the $\mathrm{HE}$ emission region is denoted by $B$. Since the magnetic field is weaker in the $\mathrm{HE}$ emission region than in the flare region with $\sim 20 \mathrm{G}$, IC scattering is the dominant radiation process. As shown in Section 3 below, the magnetic field $\lesssim 10^{-4} \mathrm{G}$ and the soft photon energy density $\sim 5 \times 10^{4} \mathrm{eV} \mathrm{cm}^{-3}$ are found to be typical values in the $\mathrm{GeV}$ emission region. For these values of the magnetic field and soft photon energy density, the radiative cooling time of electrons is longer than $\sim 4 \times 10^{9}$ s for electrons with the Lorentz factor $\lesssim 10^{5}$. Thus the cooling time is longer than the escape time and the use of the steady-state homogeneous model is justified. Here the escape time is set to be $20 r_{\gamma} / c$ in the numerical calculations.

In our flare model, we assumed the injection spectrum of electrons such as

$$
q_{\mathrm{inj}}^{f}(\gamma)=K_{e}^{f} \gamma^{-p} \exp \left(-\gamma / \gamma_{\max }^{f}\right) H\left(\gamma-\gamma_{\min }^{f}\right)
$$

where $H(z)$ is the Heaviside function. Here $K_{e}^{f}, p, \gamma_{\max }^{f}$, and $\gamma_{\min }^{f}$ are parameters. Because the flare interval is a few hours and much shorter than the dynamical timescale of the emission region $r_{\gamma} / c \sim 3 \times 10^{7} \mathrm{~s}$, we assume the continuous injection of electrons in our model. By fitting the observations, we obtained $p=1.3$ and $\gamma_{\max }^{f}=5 \times 10^{4}$ (model A in Kusunose \& Takahara (2011)). The value of $\gamma_{\min }^{f}$ was 2 . We found that the electrons in the flare region rapidly cool and obey a broken power law approximately. Because we assume that the electrons responsible for the steady $\mathrm{HE} \gamma$-rays are supplied by the flares, we use a broken power-law spectrum of electrons as the injection spectrum of electrons into the HE 
emission region. Namely,

$$
q_{\text {inj }}(\gamma)=K_{e}\left[\gamma^{-p} H\left(\gamma-\gamma_{\min }\right) H\left(\gamma_{\mathrm{br}}-\gamma\right)+\gamma^{-p_{u}} H\left(\gamma-\gamma_{\mathrm{br}}\right) H\left(\gamma_{\max }-\gamma\right)\right],
$$

where $K_{e}, \gamma_{\min }, \gamma_{\mathrm{br}}, \gamma_{\max }$, and $p_{u}$ are parameters. Note the difference of $\gamma_{\max }^{f}$ and $\gamma_{\max }$ in equations (2) and (3), respectively. The value of $K_{e}$ is determined by the injection rate per unit volume, $q_{\text {inj, }, 0}$, i.e.,

$$
q_{\mathrm{inj}, 0}=\int_{1}^{\infty} q_{\mathrm{inj}}(\gamma) d \gamma
$$

We set $\gamma_{\min }=2$ and $p=1.3$ as in a flare model (model A in Kusunose \& Takahara (2011)). In the flare region we obtained $p_{u} \sim 2.54, \gamma_{\mathrm{br}} \sim 500$, and $\gamma_{\max } \sim 2 \times 10^{5}$. To fit the Fermi data, we use $p_{u}$ and $\gamma_{\max }$ as parameters, while $\gamma_{\mathrm{br}}$ is set to be 500 . The value of $\gamma_{\mathrm{br}}$ is not important to fit the spectrum in the GeV band.

In the Galactic center region soft photons are emitted by stars, dust, and plasmas. The radiation field in the central $30^{\prime \prime}(\sim 1.2 \mathrm{pc})$ region is given in Figure 37 in Mezger et al. (1996). In their figure, the emission in $\nu<2 \times 10^{11} \mathrm{~Hz}$ is dominated by free-free emission, dust emission dominates in $2 \times 10^{11} \mathrm{~Hz} \lesssim \nu \lesssim 3 \times 10^{13} \mathrm{~Hz}$, stellar radiation in $3 \times 10^{13} \mathrm{~Hz} \lesssim$ $\nu \lesssim 2 \times 10^{16} \mathrm{~Hz}$, and hot plasmas emit X-rays in $\nu>2 \times 10^{16} \mathrm{~Hz}$. From their figure the soft photon energy density $u_{\text {soft }}$ is calculated as $9 \times 10^{-7} \mathrm{erg} \mathrm{cm}^{-3}$ or $6 \times 10^{5} \mathrm{eV} \mathrm{cm}^{-3}$. On the other hand, Hinton \& Aharonian (2007) assumed the photon energy density $5000 \mathrm{eV}$ $\mathrm{cm}^{-3}$ both in optical-UV $(3 \mathrm{eV})$ and NIR $(0.3 \mathrm{eV})$ as a radiation field model of the Galactic

center (Table 1 in their paper). They refer to the work by Davidson et al. (1992) for the soft photon energy density. Because there is uncertainty in the optical-UV energy density, we assume that the photon spectrum in the optical-UV band is approximated by a thermal radiation with temperature $T_{\text {opt-uv }}$ and energy density $u_{\text {opt-uv }}$. On the other hand, the IR spectrum is adopted from Mezger et al. (1996). In Figure1, we show an example of the soft photon spectrum used in our models. Although X-ray emission is shown in Mezger et al. (1996), we do not include X-rays as soft photons, because the Klein-Nishina effect suppresses the IC scattering of X-rays.

\section{Numerical Results}

Numerical calculations are performed with parameters such as $p_{u}, \gamma_{\max }, q_{\mathrm{inj}, 0}, B, T_{\text {opt-uv }}$, and $u_{\text {opt-uv }}$. Other parameters are fixed: $r_{\gamma}=10^{18} \mathrm{~cm}, t_{\mathrm{esc}}=20 r_{\gamma} / c, \gamma_{\min }=2, \gamma_{\mathrm{br}}=500$, and $p=1.3$. Because the HE $\gamma$-ray emission is steady during Fermi observations, the size of the emission region is greater than $\sim 1 \mathrm{lt}-\mathrm{yr}$ and we set $r_{\gamma}=10^{18} \mathrm{~cm}$. The emission spectra by electrons are not much dependent on the value of $t_{\mathrm{esc}}$, but the value of $q_{\mathrm{inj}, 0}$ is inversely proportional to $t_{\text {esc }}$. 
In Figure 2, spectral energy distributions (SEDs) are compared with the observed data. Model parameters are given in Table 1, In this figure, fixed parameters are $\gamma_{\max }=1.7 \times 10^{5}$, $B=10^{-4} \mathrm{G}$, and $T_{\text {opt-uv }}=1 \mathrm{eV}$. When $B=10^{-4} \mathrm{G}$, the gyro-radius is $1.7 \times 10^{12} \mathrm{~cm}$ for $\gamma=10^{5}$, which is short enough to confine nonthermal electrons in the HE emission region by magnetic fields. The values of $p_{u}$ and $\gamma_{\max }$ are different from our flare model for 2007 April 4 , and this may be possible because the values of $p_{u}$ and $\gamma_{\max }$ may be different from flare to flare. In the spectrum, the emission below $\sim 10^{14} \mathrm{~Hz}$ is by synchrotron radiation. Because the magnetic field is weak $\left(10^{-4} \mathrm{G}\right)$, the flux by SSC component is negligible. There are two breaks in the SEDs. Namely, breaks at $\nu_{1} \sim 10^{19} \mathrm{~Hz}$ and $\nu_{2} \sim 3 \times 10^{23} \mathrm{~Hz}$. The break at $\nu_{1}$ corresponds to the IC scattering of IR photons by electrons with $\gamma \sim \gamma_{\mathrm{br}}$. On the other hand, the break at $\nu_{2}$ is caused by IC scattering of optical-UV photons by electrons with $\gamma \sim \gamma_{\mathrm{br}}$. We assumed various values of $p_{u}$ in Figure 2. The spectral shape at $\nu \gtrsim \nu_{2}$ does not depend on the value of $p_{u}$, because the values of $u_{\text {opt-uv }}$ and $q_{\text {inj, } 0}$ are adjusted to fit the flux of $\mathrm{GeV} \gamma$-rays. It is to be noted that the photons with $\nu \gtrsim \nu_{2}$ are produced by IC scattering of optical-UV photons by electrons with $\gamma \sim \gamma_{\max }$ and that the scattering occurs in the Klein-Nishina regime.

It is noted that the radio emission of our models exceed that of $\mathrm{Sgr} \mathrm{A}^{*}$ for $\nu<10^{10} \mathrm{~Hz}$, but the model emission should be compared with that of 1 pc region from Sgr $A^{*}$. This is shown in Figure 4 by a dotted line. Some parameter values yield excess emission at $\lesssim 10^{9}$ Hz. This excess is, however, avoided if the magnetic field is weaker and the difference in the magnetic field does not affect the $\mathrm{GeV}$ emission spectrum. In Figure 2 we fixed $B=10^{-4} \mathrm{G}$ to compare the $\mathrm{GeV}$ spectra of various models.

When the GeV spectrum is fitted with different values of $p_{u}$, the soft photon energy density in the optical-UV band should be adjusted with $p_{u}$. Model A6 is presented to show the effect of the soft photons in the optical-UV band. The parameters of A6 is different from those of model A3 only in $u_{\text {opt-uv }}$. The HE emission of A6 is produced mainly by IC scattering of IR photons. It is found that the soft photons in the optical-UV band are important to account for emission at $\nu \gtrsim 10^{23} \mathrm{~Hz}$. The effect of IC scattering of optical-UV photons in the $\mathrm{GeV}$ band becomes apparent for $u_{\text {opt-uv }} \gtrsim 10^{4} \mathrm{eV} \mathrm{cm}^{-3}$.

The electron kinetic energy density, $u_{\text {kin }}$, is different from model to model. In model A3, the value of $u_{\text {kin }}$ is $\sim 4.7 \times 10^{-7} \mathrm{erg} \mathrm{cm}^{-3}$. The electron kinetic energy contained in the emission region is $\sim 2.0 \times 10^{48} \mathrm{erg}$ and the energy injection rate of electrons is $1.3 \times 10^{39}$ $\operatorname{erg~} \mathrm{s}^{-1}$. (Note that this value is inversely proportional to $t_{\mathrm{esc}}$.) This injected energy is mostly possessed by electrons with $\gamma<\gamma_{\mathrm{br}}$, and the electrons emitting the GeV $\gamma$-rays have only a fraction of the injected energy: the electrons with $\gamma>\gamma_{\mathrm{br}}$ contribute to the energy density only $\sim 1 \%$, when $p_{u}=2.7$. That is, the energy injection rate $\lesssim 10^{37} \mathrm{erg} \mathrm{s}^{-1}$ is used 
to emit $\mathrm{GeV} \gamma$-rays. This number is just consistent with our flare model, if the radiation efficiency during flares is low and most of the kinetic energy of electrons is transported to the HE emission region. In our flare model, the emission efficiency is found to be $\sim 10 \%$ by numerical calculations. The observed flare luminosity of $\sim 10^{36} \mathrm{erg} \mathrm{s}^{-1}$ means that $\sim 10^{37}$ $\mathrm{erg} \mathrm{s}^{-1}$ is injected into the flare emission region, and that the most of the energy is directed to the environment without emission. If the duty cycle of the flares is $10 \%$ as observed, $10 \%$ of $10^{37} \mathrm{erg} \mathrm{s}^{-1}$, i.e., $10^{36} \mathrm{erg} \mathrm{s}^{-1}$, is directed to the steady $\mathrm{GeV}$ emission. Considering various uncertainties regarding the flare models and $\mathrm{GeV}$ emission region, the energetics of the current model is acceptable. It is suggestive that the luminosities of the flare emission and the steady $\mathrm{GeV}$ emission are both $\sim 10^{36} \mathrm{erg} \mathrm{s}^{-1}$.

In Figure 3, SEDs are shown for $p_{u}=2.7$ and various values of soft photon parameters. The parameter values are given in Table 2, The optical-UV emission with $T_{\text {opt-uv }}=3 \mathrm{eV}$ is assumed for models B1 and B2. When the value of $T_{\text {opt-uv }}$ is larger, larger values of $u_{\text {opt-uv }}$ (B1) or $q_{\text {inj, } 0}$ (B2) are required. We found numerically that $u_{\text {opt-uv }}$ should be $\gtrsim 10^{5} \mathrm{eV} \mathrm{cm}^{-3}$ for $T_{\text {opt-uv }} \gtrsim 3 \mathrm{eV}$ to fit the observed $\mathrm{GeV}$ spectrum. Larger values of $q_{\mathrm{inj}, 0}$ results in a poor fit as shown by model B2 in Figure 3 .

In Figure 4, SEDs for $p_{u}=2.6$ are compared with the emission expected from the central 30" given in Mezger et al. (1996). Our models are calculated with $B=10^{-4} \mathrm{G}$ (solid line) and $3 \times 10^{-4} \mathrm{G}$ (dash-dotted line). The soft photon energy density is larger than the magnetic energy density and the effect of the difference in the magnetic field appears only in the synchrotron radiation. Synchrotron emission exceeds the radio flux observed in the central $30^{\prime \prime}$ when $B>10^{-4} \mathrm{G}$. Our model thus sets constraint on the average strength of the magnetic field in the central $1 \mathrm{pc}$ region.

In Figure 5, we show a model with $\gamma_{\max }=6 \times 10^{7}$ to see the possibility of $\mathrm{TeV}$ emission by our model. Here we assumed a smaller value of the electron injection rate than for the $\mathrm{GeV}$ emission models, i.e., $q_{\mathrm{inj}, 0}=3 \times 10^{-13} \mathrm{~s}^{-1}$ and this corresponds to the energy injection rate of $9.8 \times 10^{37} \mathrm{erg} \mathrm{s}^{-1}$. This is much smaller than for model A3 (dashed line), whose energy injection rate is $1.3 \times 10^{39} \mathrm{erg} \mathrm{s}^{-1}$. Since the maximum Lorentz factor of electrons is much larger than our flare model, efficient acceleration of electrons must occur during the propagation from the flare region to the $\mathrm{HE}$ emission region. Alternatively, electrons from other sources such as pulsar wind nebulae are responsible for the TeV emission. This kind of model was presented by Atoyan \& Dermer (2004) and Hinton \& Aharonian (2007). 


\section{DISCUSSION}

We have demonstrated that the GeV $\gamma$-ray spectrum from Sgr A* obtained by Fermi is well fitted by IC scattering in a region with a radius of $10^{18} \mathrm{~cm}$, when soft photons are supplied by stars and dust. We assumed that the nonthermal electrons are supplied by flare events near the central black hole, which are often observed in the NIR and X-ray bands. To fit the Fermi data, we assumed electrons with $p_{u} \gtrsim 2.6$ and $\gamma_{\max } \gtrsim 10^{5}$. The success of our HE emission model, in turn, supports our NIR/X-ray flare model.

We found that the magnetic field in the region within $\sim 1$ pc from the central black hole is $\lesssim 10^{-4} \mathrm{G}$ because synchrotron emission exceeds the observed radio flux in the central 1 pc region. The value of $\sim 10^{-4} \mathrm{G}$ is also consistent with another constraint given by Crocker et al. (2011).

Because the distance that nonthermal electrons travel from the flare region to the HE emission region is $\sim 10^{18} \mathrm{~cm}$, the emission by those electrons during transport is to be considered. When electrons are close to a flare region, they emit radiation mostly by synchrotron emission. Because the soft photon energy density in the HE emission region is $\sim 5 \times 10^{4} \mathrm{eV}$ $\mathrm{cm}^{-3}$, synchrotron radiation dominates over IC scattering in regions with $B \gtrsim 10^{-3} \mathrm{G}$. Since the magnetic field of the flare emission region is $\sim 20 \mathrm{G}$ and that in the HE emission region is $\sim 10^{-4} \mathrm{G}$, synchrotron emission dominates over IC scattering in a region within $\sim 10^{17}$ $\mathrm{cm}$ from the central black hole, if the electron density is constant and the magnetic field decreases as $B \propto r^{-1}$, where $r$ is the distance from the central black hole. Then the volume of the synchrotron dominant region is smaller than that of the HE emission region by a factor of $10^{-3}$. When the electron density decreases more rapidly than $r^{-2}$, the contribution of the central region to the synchrotron emission is significant. That is, a large flux of observed $\mathrm{GeV}$ emission suggests that nonthermal electrons do not follow a wind-like flow. On the other hand, if $B \propto r^{-1}$ is assumed as above, the decrease of the electron kinetic energy by synchrotron cooling is small. For example, if the Lorentz factor of escaping electrons from a flare region at $r \sim 10^{13} \mathrm{~cm}$ is $\gamma=10^{5}$, it decreases to $\sim 2 \times 10^{4}$ at $r \sim 10^{17} \mathrm{~cm}$ and is almost constant for $r \gtrsim 10^{17} \mathrm{~cm}$. Here we assumed that electrons propagate at speed of light. If the magnetic field decreases more rapidly than $r^{-1}$, this decrease in $\gamma$ becomes slower. Therefore, the effect of radiative cooling during electron transport from flare regions to the HE emission region is not important.

We assumed that the size of the $\mathrm{HE}$ emission region, $r_{\gamma}$, is $10^{18} \mathrm{~cm}$ in this work. Observationally there is no strong constraint on the value of $r_{\gamma}$, except that the HE emission is consistent with no time variation during Fermi observation. One possible constraint is that $r_{\gamma} \lesssim c t_{\mathrm{IC}}$ to fill the region of $r_{\gamma}$ with electrons with $\gamma$ up to $\gamma_{\max }$, where $t_{\mathrm{IC}}$ is the IC cooling time. This sets the upper limit of $r_{\gamma} \lesssim 10^{20}\left(\gamma / 10^{5}\right)^{-1} \mathrm{~cm}$ for $u_{\text {soft }} \sim 5 \times 10^{4} \mathrm{erg}$ 
$\mathrm{s}^{-1}$. On the other hand, the $\mathrm{GeV}$ emission by IC scattering in a more compact region near the central black hole is unlikely as follows. In a region near the black hole, the soft photon source is most likely the RIAF and the magnetic filed is approximately $\sim 1 \mathrm{G}$. When the $\mathrm{GeV}$ emission is by IC scattering off soft photons, there is a constraint such that $u_{B}<u_{\text {soft }}$, where $u_{B}$ is the energy density of the magnetic field. This results in

$$
r_{\gamma} \lesssim \frac{1}{B}\left(\frac{\epsilon L_{\mathrm{bol}}}{c}\right)^{1 / 2} \sim 6 \times 10^{12} \epsilon^{1 / 2}\left(\frac{1 \mathrm{G}}{B}\right)\left(\frac{L_{\mathrm{bol}}}{10^{36} \mathrm{erg} \mathrm{s}^{-1}}\right)^{1 / 2} \mathrm{~cm},
$$

where $\epsilon$ is the scattered energy fraction of RIAF luminosity. When $B=1 \mathrm{G}, \epsilon<1$, and $L_{\mathrm{bol}}=10^{36} \mathrm{erg} \mathrm{s}^{-1}$, we obtain $r_{\gamma}<10^{13} \mathrm{~cm}$, which size is comparable with a flare emission region of our leptonic flare model. Then the HE emission region may exhibit time variation with timescale of $r_{\gamma} / c \sim 300 \mathrm{~s}$, contrary to the observed steady GeV emission.

Because $\mathrm{TeV} \gamma$-rays are absorbed by $e^{+} e^{-}$pair production in collisions with soft photons, the soft photon density should be small enough to avoid the absorption to account for the $\mathrm{TeV}$ emission observed by HESS. The optical depth of the absorption is given by $\tau_{\gamma \gamma} \sim 0.3 \sigma_{\mathrm{T}} r_{\gamma} n_{s}$ near the threshold, where $\sigma_{\mathrm{T}}$ is the Thomson cross section and $n_{s}$ is the soft photon density. Since $r_{\gamma}=10^{18} \mathrm{~cm}, n_{s}<5 \times 10^{6} \mathrm{~cm}^{-3}$ is required for $\tau_{\gamma \gamma}<1$. As shown in Figure 1, the soft photon spectrum has two peaks at IR and optical-UV bands. The photon density at the IR peak of $\nu \sim 2 \times 10^{13} \mathrm{~Hz}$ is $\sim 2 \times 10^{5} \mathrm{~cm}^{-3}$ and this gives $\tau_{\gamma \gamma} \sim 4 \times 10^{-2}$. At $\nu \sim 10^{15}$ $\mathrm{Hz}$, on the other hand, $n_{s} \sim 9 \times 10^{3} \mathrm{~cm}^{-3}$ for $T_{\text {opt-uv }}=1 \mathrm{eV}$ and $u_{\text {opt-uv }}=5 \times 10^{4} \mathrm{erg} \mathrm{cm}^{-3}$. The optical depth is then $\tau_{\gamma \gamma} \sim 2 \times 10^{-3}$. As mentioned in Section 3, $T_{\text {opt-uv }} \gtrsim 3 \mathrm{eV}$ needs

$u_{\text {opt-uv }} \gtrsim 10^{5}$ to fit the $\mathrm{GeV}$ emission. Thus when the value of $T_{\text {opt-uv }}$ is larger, the central region becomes opaque for $\mathrm{TeV} \gamma$-rays, contrary to the observations of $\mathrm{TeV} \gamma$-rays.

This work has been partially supported by KAKENHI (F.T.: 20540231) from the Ministry of Education, Culture, Sports, Science and Technology of Japan.

\section{REFERENCES}

Abdo, A. A., Ackermann, M., Ajello, M., et al. 2009, ApJS, 183, 46

Aharonian, F., Akhperjanian, A. G., Anton, G., et al. 2009, A\&A, 503, 817

Aharonian, F., Akhperjanian, A. G., Aye, K.-M., et al. 2004, A\&A, 425, L13

Aharonian, F., Akhperjanian, A. G., Barres de Almeida, U., et al. 2008, A\&A, 492, L25

Aharonian, F., Akhperjanian, A. G., Bazer-Bachi, A. R., et al. 2006, Phys. Rev. Lett., 97, 221102 
Aharonian, F. \& Neronov, A. 2005, ApJ, 619, 306

Albert, J., Aliu, E., Anderhub, H., et al. 2006, ApJ, 638, L101

Atoyan, A. \& Dermer, C. D. 2004, ApJ, 617, L123

Baganoff, F. K., Maeda, Y., Morris, M., et al. 2003, ApJ, 591, 891

Baganoff, F. K. 2003, BAAS, 35, 606

Ballantyne, D. R., Melia, F., Liu, S., \& Crocker, R. M. 2007, ApJ. 657, L13

Chernyakova, M., Malyshev, D., Aharonian, F. A., Crocker, R. M., \& Jones, D. I. 2011, ApJ, 726, 60

Cohen-Tanugi, J., Pohl, M., Tibolla, O., \& Nuss, E. 2009, Proc. 31st Int. Cosmic Ray Conf., Łódź, 645 (http://icrc2009.uni.lodz.pl/proc/pdf/icrc0645.pdf)

Crocker, R. M., Jones, D. I., Aharonian, F., et al., 2011, MNRAS, 413, 763

Davidson, J. A., Werner, M. W., Wu, X., et al. 1992, ApJ, 387, 189

Dodds-Eden, K., Gillessen, S., Fritz, T. K., et al. 2011, ApJ, 728, 37

Dodds-Eden, K., Porquet, D., Trap, G., et al. 2009, ApJ, 698, 676

Eckart, A., Baganoff, F. K., Sch" odel, R., et al. 2006, A\&A, 450, 535

Eisenhauer, F., Schödel, R., Genzel, R., et al. 2003, ApJ, 597, L121

Falcke, H., Goss, W. M., Matsuo, H., et al. 1998, ApJ, 499, 731

Genzel, R., Eisenhauer, F., \& Gillessen, S. 2010, Rev. Mod. Phys., 82, 3121

Genzel, R., Schödel, R., Ott, T., et al. 2003, Nature, 425, 934

Falcke, H. \& Markoff, S. 2000, A\&A, 362, 113

Ghez, R., Salim, S., Weinberg, N. N., et al. 2008, ApJ, 689, 1044

Gillessen, S., Eisenhauer, F., Fritz, T. K., et al. 2009a, ApJ, 707, L114

Gillessen, S., Eisenhauer, F., Trippe, S., et al. 2009b, ApJ, 692, 1075

Hinton, J. A. \& Aharonian, F. A. 2007, ApJ, 657, 302

Kosack, K., Badran, H. M., Bond, I. H., et al. 2004, ApJ, 608, L97 
Kusunose, M. \& Takahara, F. 2011, ApJ, 726, 54

Markoff, S., Falcke, H., Yuan, F., \& Biermann, P. L. 2001, A\&A, 379, L13

Melia, F. 2007, The Galactic Supermassive Black Hole (Princeton, NJ: Princeton Univ. Press)

Mezger, P. G., Duschl, W. J., \& Zylka, R. 1996, A\&AR, 7, 289

Moskalenko, I. V., Porter, T. A., \& Strong, A. W. 2006, ApJ, 640, L155

Porquet, D., Grosso, N., Predehl, P., et al. 2008, A\&A, 488, 549

Porter, T. A. \& Strong, A. W. 2005, Proc. 29th Int. Cosmic Ray Conf., Pune, 4, 77

Rolland, L. \& Hinton, J. 2005, Proc. 29th Int. Cosmic Ray Conf., Pune, 4, 109

Trap, G., Goldwurm, A., Dodds-Eden, K., et al. 2011, A\&A, 528, A140

Tsuchiya, K., Enomoto, R., Ksenofontov, L. T., et al. 2004, ApJ, 606, L115

Yuan, F., Quataert, E., \& Narayan, R. 2003, ApJ, 598, 301

Zhan, J.-L., Bi, X.-J., \& Hu, H.-B. 2006, A\&A, 449, 641

Zhao, J.-H., Young, K. H., Herrnstein, R. M., et al. 2003, ApJ, 586, L29

Zylka, R., Mezger, P. G., Ward-Thompson, D., Duschl, W. J., \& Lesch, H. 1995, A\&A, 297, 83 


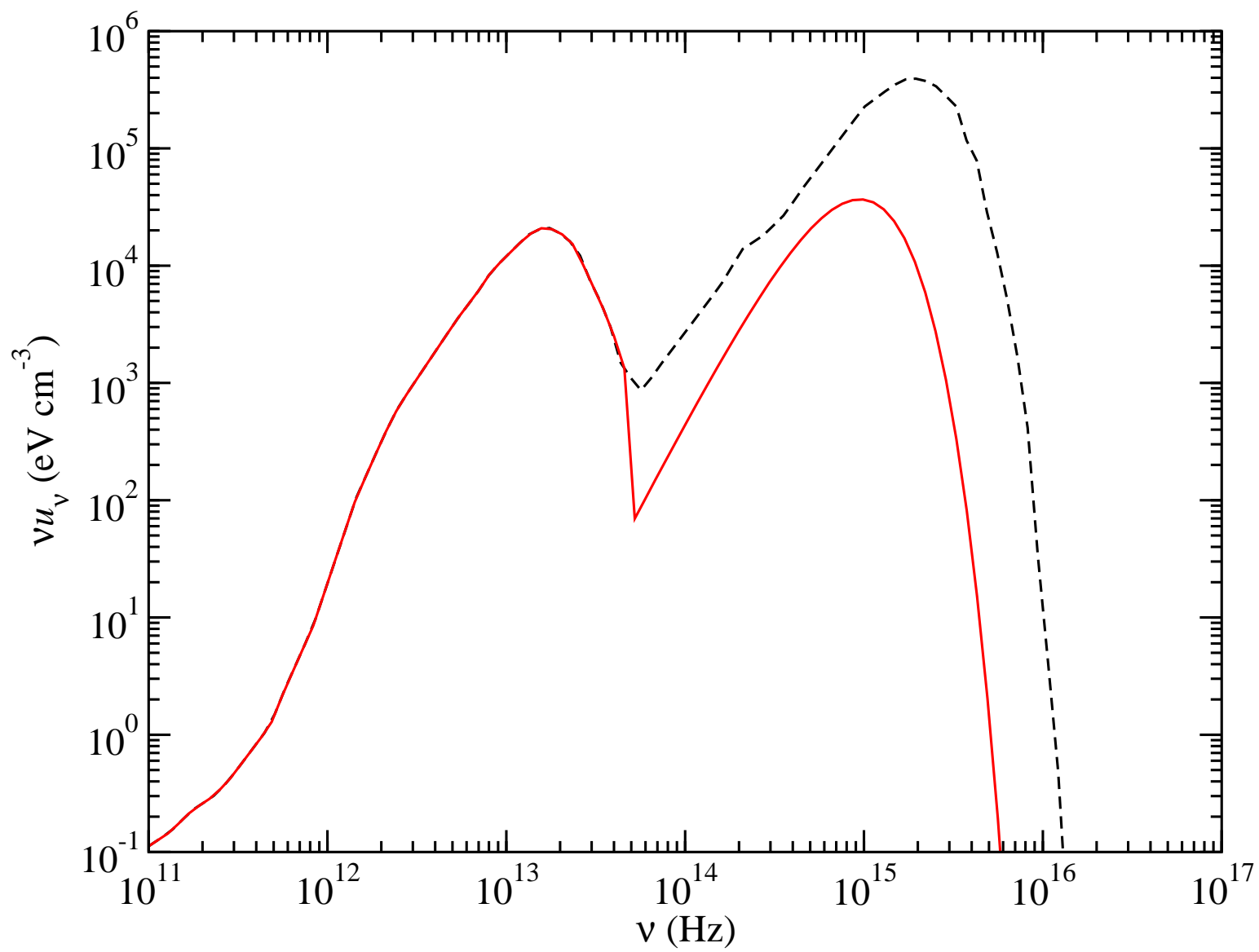

Fig. 1. - Soft photon energy density spectrum in the central region. The dashed line is adopted from Mezger et al. (1996). Our model (solid curve) uses their IR emission spectrum but the optical-UV spectrum is calculated as a blackbody with parameters $T_{\text {opt-uv }}$ and $u_{\text {opt-uv }}$. The solid curve is calculated for $T_{\text {opt-uv }}=1 \mathrm{eV}$ and $u_{\text {opt-opt }}=5 \times 10^{4} \mathrm{eV} \mathrm{cm}^{-3}$. 


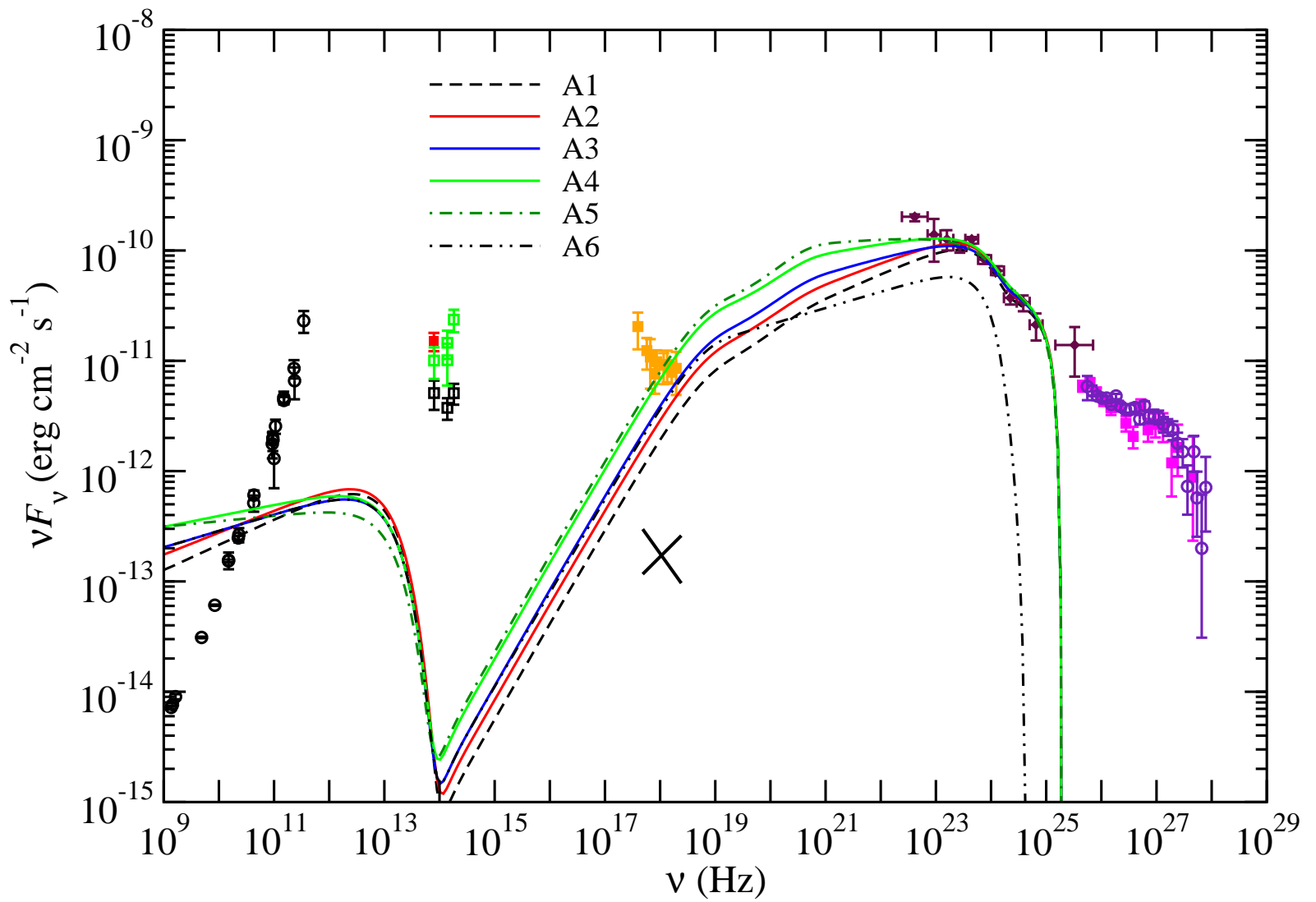

Fig. 2.- SEDs for various values of $p_{u}$. Here $\gamma_{\max }=1.7 \times 10^{5}, B=10^{-4} \mathrm{G}$, and $T_{\text {opt-uv }}=1$ $\mathrm{eV}$. The value of $u_{\text {opt-uv }}$ is changed to fit the data. Model parameters are given in Table 1 1 . The data in the range $10^{22}$ to $3 \times 10^{25} \mathrm{~Hz}$ are obtained by Fermi (Chernyakova et al. 2011). $\mathrm{TeV}$ emission data are from Aharonian et al. (2006) (filled squares) and Aharonian et al. (2009) (open circles). Radio to submillimeter measurements are for the quiescent state (Markoff et al. 2001; Zhao et al. 2003) (open circles). IR data in the quiescent state are from Genzel et al. (2003). The X-ray data in the quiescent state are from Baganoff et al. (2003). The flaring state in NIR (filled square) is taken from Dodds-Eden et al. (2009). The X-ray flare data (filled squares) are from Porquet et al. (2008). 


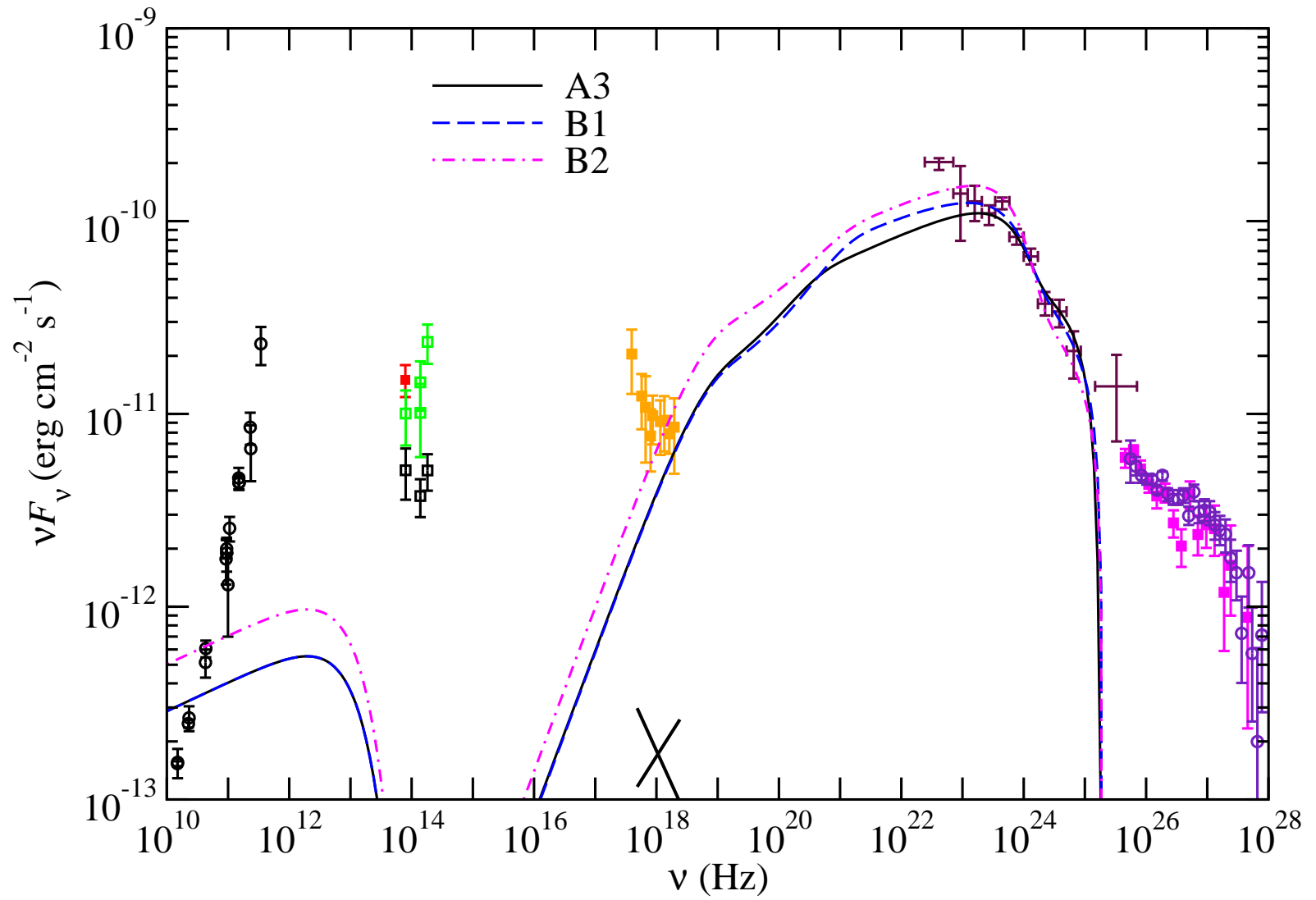

Fig. 3.- SEDs with $p_{u}=2.7$ for different soft photon parameters. The parameter values are given in Table 2 , 


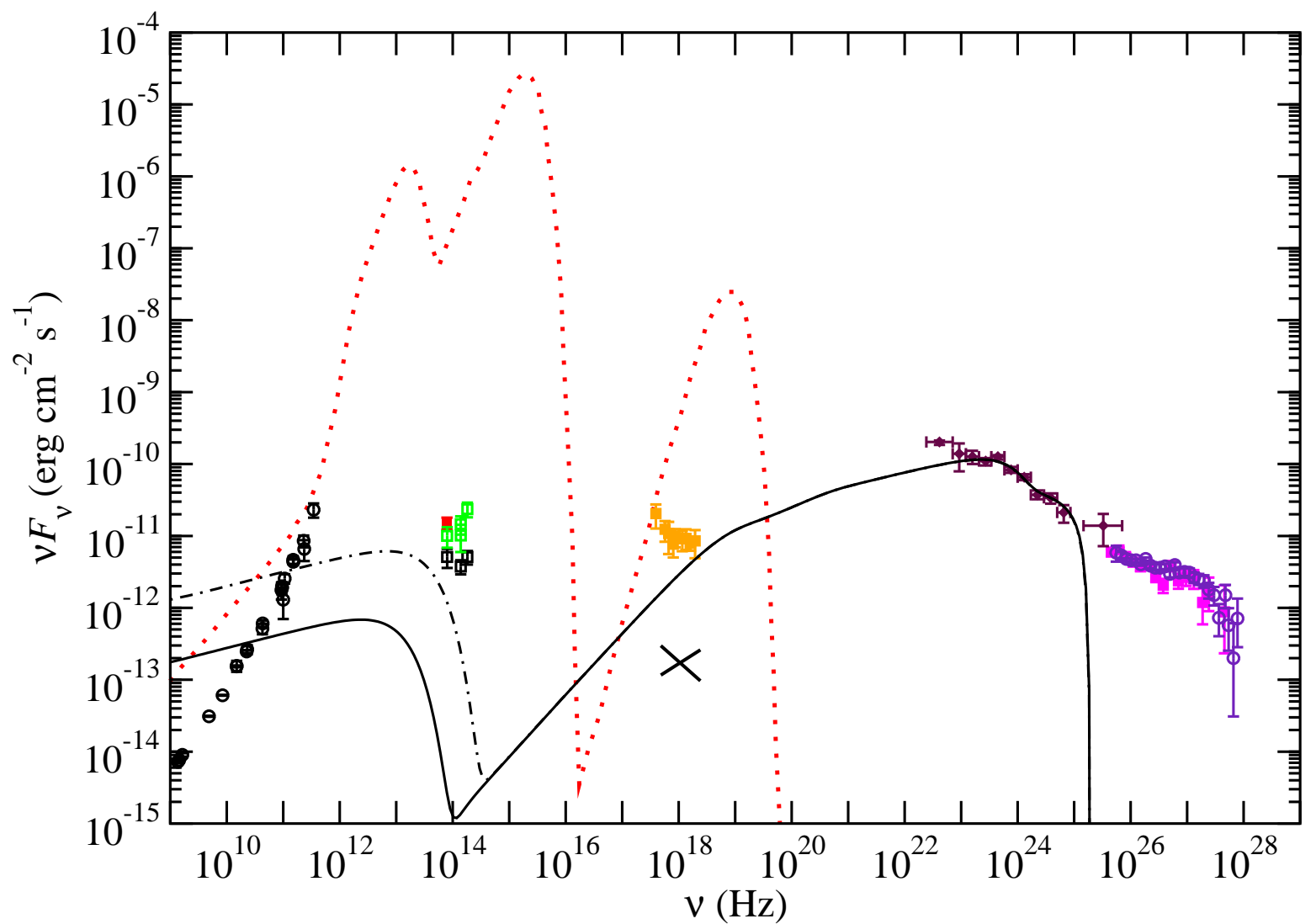

Fig. 4.- Effect of magnetic field. Model SEDs with $B=10^{-4} \mathrm{G}$ (solid) and $B=3 \times 10^{-4}$ $\mathrm{G}$ (dash-dotted) are compared with the emission (dotted) in the central region $\lesssim 1.2 \mathrm{pc}$ (Mezger et al. 1996). Here, $p_{u}=2.6, \gamma_{\max }=1.7 \times 10^{5}, T_{\text {opt-uv }}=1 \mathrm{eV}$, and $u_{\text {opt-uv }}=4 \times 10^{4}$ $\mathrm{eV} \mathrm{cm}^{-3}$ are assumed to calculate our models. 


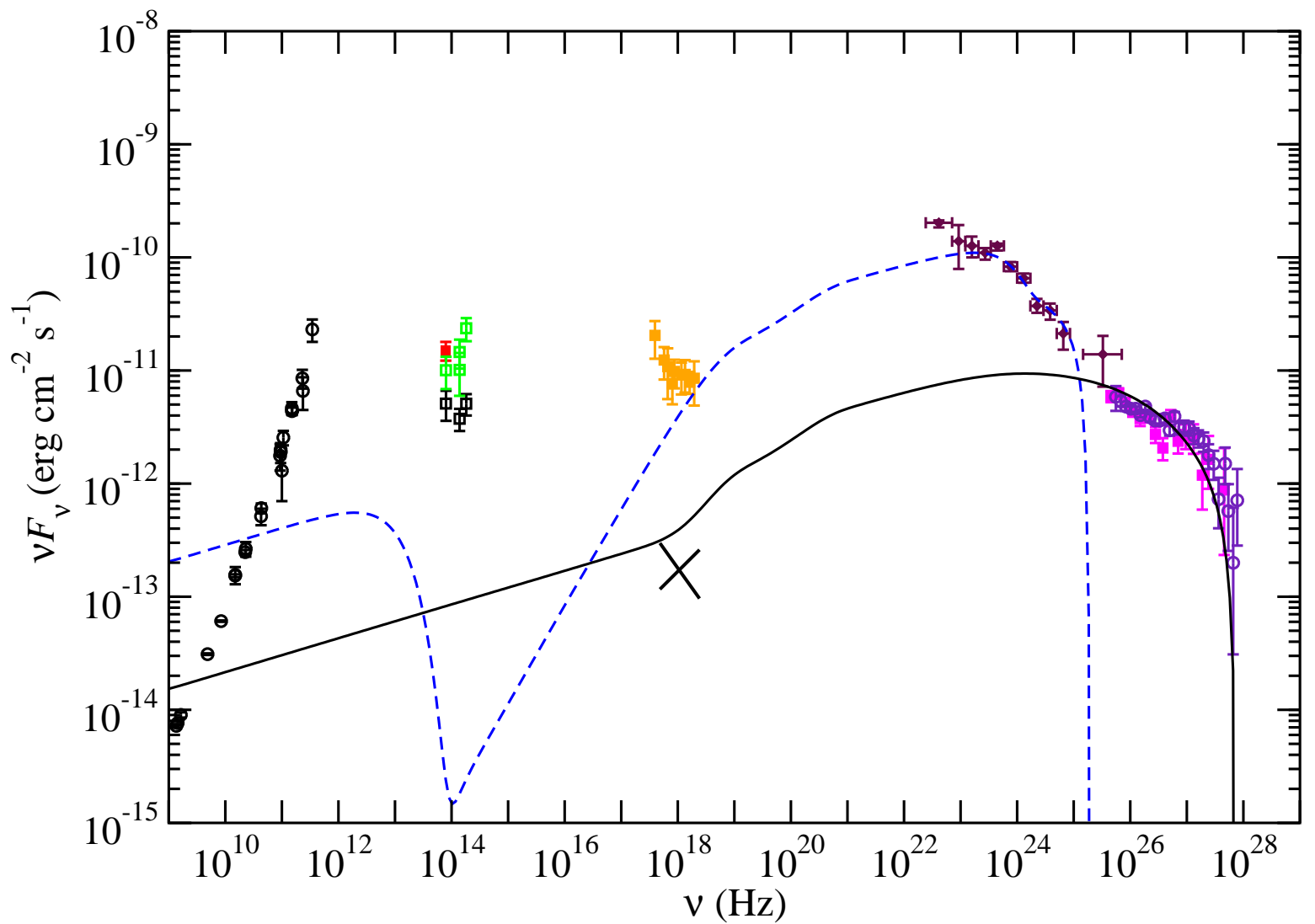

Fig. 5. - Emission model of $\mathrm{TeV} \gamma$-rays. The solid line is calculated for $\gamma_{\max }=6 \times 10^{7}$. Here, $p_{u}=2.7, B=10^{-4} \mathrm{G}, T_{\text {opt-uv }}=1 \mathrm{eV}$, and $u_{\text {opt-uv }}=5 \times 10^{4} \mathrm{eV} \mathrm{cm}^{-3}$ are assumed. Model A3 with $\gamma_{\max }=1.7 \times 10^{5}$ is shown by a dashed line for comparison. 
Table 1. Parameters

\begin{tabular}{cccc}
\hline \hline Model & $p_{u}$ & $\begin{array}{c}u_{\text {opt-uv }} \\
\left(\mathrm{eV} \mathrm{cm}{ }^{-3}\right)\end{array}$ & $\begin{array}{c}q_{\text {inj, },} \\
\left(\mathrm{cm}^{-3} \mathrm{~s}^{-1}\right)\end{array}$ \\
\hline A1 & 2.54 & $4 \times 10^{4}$ & $2 \times 10^{-12}$ \\
A2 & 2.6 & $4 \times 10^{4}$ & $3 \times 10^{-12}$ \\
A3 & 2.7 & $5 \times 10^{4}$ & $4 \times 10^{-12}$ \\
A4 & 2.8 & $5 \times 10^{4}$ & $7 \times 10^{-12}$ \\
A5 & 2.9 & $7 \times 10^{4}$ & $8 \times 10^{-12}$ \\
A6 & 2.7 & 1 & $4 \times 10^{-12}$ \\
\hline
\end{tabular}

Note. - All models assume $p=1.3$, $\gamma_{\min }=2, \gamma_{\max }=1.7 \times 10^{5}, r_{\gamma}=10^{18} \mathrm{~cm}$, $B=10^{-4} \mathrm{G}$, and $T_{\text {opt-uv }}=1 \mathrm{eV}$. 
Table 2. Parameters

\begin{tabular}{cccc}
\hline \hline Model & $\begin{array}{c}T_{\text {opt-uv }} \\
(\mathrm{eV})\end{array}$ & $\begin{array}{c}u_{\text {opt-uv }} \\
\left(\mathrm{eV} \mathrm{cm} \mathrm{cm}^{-3}\right)\end{array}$ & $\begin{array}{c}q_{\text {inj, } 0} \\
\left(\mathrm{~cm}^{-3} \mathrm{~s}^{-1}\right)\end{array}$ \\
\hline B1 & 3 & $9 \times 10^{4}$ & $4 \times 10^{-12}$ \\
B2 & 3 & $4 \times 10^{4}$ & $7 \times 10^{-12}$ \\
\hline
\end{tabular}

Note. - All models assume $p=1.3, \gamma_{\min }=$ $2, \gamma_{\max }=1.7 \times 10^{5}, r_{\gamma}=10^{18} \mathrm{~cm}$, and $B=$ $10^{-4} \mathrm{G}$. 